\title{
Role of PGC-1a in Sarcopenia: Etiology and Potential Intervention - A Mini-Review
}

\author{
Li Li Ji Chounghun Kang \\ Laboratory of Physiological Hygiene and Exercise Science, School of Kinesiology, University of Minnesota, \\ Minneapolis, Minn., USA
}

\section{Key Words}

Aging $\cdot$ Mitochondria $\cdot$ Muscle $\cdot$ PGC-1a $\cdot$ Sarcopenia .

Signaling

\begin{abstract}
Sarcopenia is age-associated deterioration of muscle mass and function caused by a wide scope of physiological and pathological changes ranging from hormonal disorders to loss of subcellular homeostasis. Recent research indicates that mitochondrial dysregulation with advanced age plays a central role in the development of sarcopenia due to the multifactorial functions of this organelle in energy supply, redox regulation, crosstalk with nuclear gene expression and apoptosis. In order to fulfill these roles, it is crucial that mitochondria maintain their own structural and functional integrity through biogenesis, antioxidant defense, fusion/fission dynamics and autophagy (mitophagy). Unfortunately, mitochondria undergo age-associated changes that compromise the above-mentioned properties that eventually contribute to the development of sarcopenia. The peroxisome proliferator-activated receptor- $\gamma$ coactivator-1a (PGC-1a) is involved in the transcriptional regulation of numerous nuclear and mitochondrial gene products participating in the cellular events that control muscle mass and function. Thus, it is not surprising that maintaining an optimal intracellular PGC1a level and signaling activity is crucial in protecting the muscle from many degradative and destructive processes, such as proteolysis, oxidative damage, inflammation, uncontrolled autophagy and apoptosis. Physical exercise is a pow-
\end{abstract}

erful stimulus to PGC-1a expression and signaling. Recent research indicates that PGC-1a-controlled mitochondrial biogenesis is not limited by old age per se and that elderly individuals can still benefit from increased muscular activity in terms of skeletal muscle health that ultimately contributes to quality of life in old age.

(c) 2014 S. Karger AG, Basel

\section{Introduction}

Sarcopenia is a degenerative state of skeletal muscle characterized by significant loss of muscle mass and function in later life, although the process may start earlier when a person passes middle age. This highly prevalent syndrome is regarded as one of the top health threats to the aging population due to its predictable relevance to frailty, immobility, disability, hospitalization and mortality [1]. Thus, to gain a thorough understanding of the cellular mechanisms that govern the pathogenesis of sarcopenia has become one of the top priorities of fundamental as well as clinical research during the past 2 decades.

Numerous pathways have been identified to have potential influences on the degeneration of muscle fibers, including hormonal, neural, metabolic and molecular signals that alter their input during aging $[2,3]$. There is increasing evidence that mitochondrial abnormality plays a crucial role due to their multifaceted functions within the cell $[4,5]$. First, mitochondria provide the bulk of ATP production, which becomes a vital resource for 
aging myocytes to maintain viability. Second, mitochondria regulate the redox homeostasis such that reactive oxygen species (ROS) are not overly produced and/or accumulated to activate catabolic pathways (such as proteolysis) and inflammation. Third, mitochondrion is a vital organelle that participates in the regulation of apoptosis, a highly relevant cellular event in senescent muscle. Finally, the fact that mitochondria contain their own DNA makes it possible to coordinate with nuclear DNA for necessary signaling and remodeling of muscle cells in response to reduced load, blood flow, nutrition and other changes occurring in advanced age. It is well known that loss of mitochondrial number and functional activity is the hallmark of aging and age-related mortality, such as neurodegenerative diseases, diabetes and certain types of cancer [6]. Thus, it is not surprising that much of the current research on sarcopenia has been focused on this critical organelle. Readers are referred to more detailed reviews on the role of mitochondria in sarcopenia $[2-5,7]$.

Over the past decade, an overwhelming volume of literature has indicated that the peroxisome proliferatoractivated receptor- $\gamma$ coactivator-1 $\alpha$ (PGC- $1 \alpha$ ) plays a critical role in the regulation of mitochondrial structural and functional integrity during the pathogenic processes of muscle atrophy and aging $[8,9]$. As a master regulator of mitochondrial integrity, function and biogenesis, as well as a key player in other cellular events, such as antioxidant defense, muscle fiber transformation, inflammation, fusion and fission, and insulin signaling, PGC-1a controls a wide array of cellular mechanisms that directly or indirectly affect the maintenance of myocyte number, protein level and functionality during aging. On the other hand, aging is known to alter several important pathways that may affect PGC-1 $\alpha$ expression and activity. Thus, understanding how PGC-1 $\alpha$ changes its cellular content and posttranslational activity, and how it interacts with other signaling pathways that have a significant impact on muscle protein synthesis and degradation in aging muscle, will provide valuable insights into the mechanism of sarcopenia and the identification of potential targets for treatment.

\section{Role of Mitochondria in Sarcopenia}

\section{Age-Associated Changes in Skeletal Muscle}

The progressive loss of muscle mass and strength is one of the hallmarks of the aging process. Previous evidence has shown a $3 \sim 10 \%$ decline of muscle mass per decade after the age of 25 years, mainly due to decreases in skeletal muscle cross-sectional area with age [10]. There was also clear evidence that the muscles of older people (65-83 years of age) contain less contractile tissue and more noncontractile tissues (fat and connective tissue) compared with young controls [11]. Thus, a greater percentage of noncontractile tissues results in a decreased force production.

Both intrinsic and extrinsic factors contribute to the development of sarcopenia [3]. Intrinsic factors include: (i) a deterioration of protein synthesis required to replace the wasting muscle; (ii) decreased androgen levels, such as testosterone, GH and IGF that stimulate protein synthesis; (iii) increased proteolysis and autophagy due to ROS generation and inflammation; (iv) decline of motor nerve stimulation, including the dysfunction of the neuromuscular junction (NMJ) program, and (v) enhanced apoptosis and decreased satellite and stem cell activities. Extrinsic factors include: (i) decreased physical activity and, hence, contractile frequency and load; (ii) altered nutrition due to dietary habit change; (iii) diseases that affect muscle well-being (such as cancer, diabetes and cardiovascular diseases), and (iv) medicine, smoking and drug uses that alter muscle protein homeostasis. It is noteworthy that during aging life style changes could affect both intrinsic and extrinsic factors that eventually impact on muscle metabolism and integrity [12]. Furthermore, traumatic events, such as heart attack, stroke and bone fracture due to falls that become more common among elderly people can cause dramatic changes in many of the above-mentioned factors, resulting in loss of muscle mass and function. Thus, sarcopenia is not a disease but a syndrome associated with advanced age. In the wild, older animals usually die of starvation, predication and sickness before significant muscle loss due to age sets in. In underdeveloped countries and regions of the world sarcopenia is often masked by malnutrition, diseases and early death. In industrialized countries with improved longevity and steady removal of some of the negative regulators, finding the critical pathogenic links to the deterioration of muscle mass and functionality has become an increasing challenge for researchers.

\section{Mitochondrial Dysfunction with Ageing}

Contemporary research clearly indicates that mitochondrial deterioration is central to the pathology of age-associated changes in cellular structure and function of many organs and tissues, including the skeletal muscle $[4,5]$. Mitochondria in skeletal muscle elicit rapid and characteristic changes as a consequence of manipulations of muscle use and environmental conditions. Losses of mitochondrial content and functional 
Fig. 1. A brief overview of ROS-induced pathways that affect mitochondrial homeostasis and the role of PGC-1 $\alpha$ in protecting the senescent muscle during sarcopenia. By inducing antioxidant enzymes and inhibiting $\mathrm{NF} \kappa \mathrm{B}, \mathrm{PGC}-1 \alpha$ reduces ROS and inflammation, which are the main negative regulators of mitochondrial homeostasis. PMN = Polymorphoneutrophil.

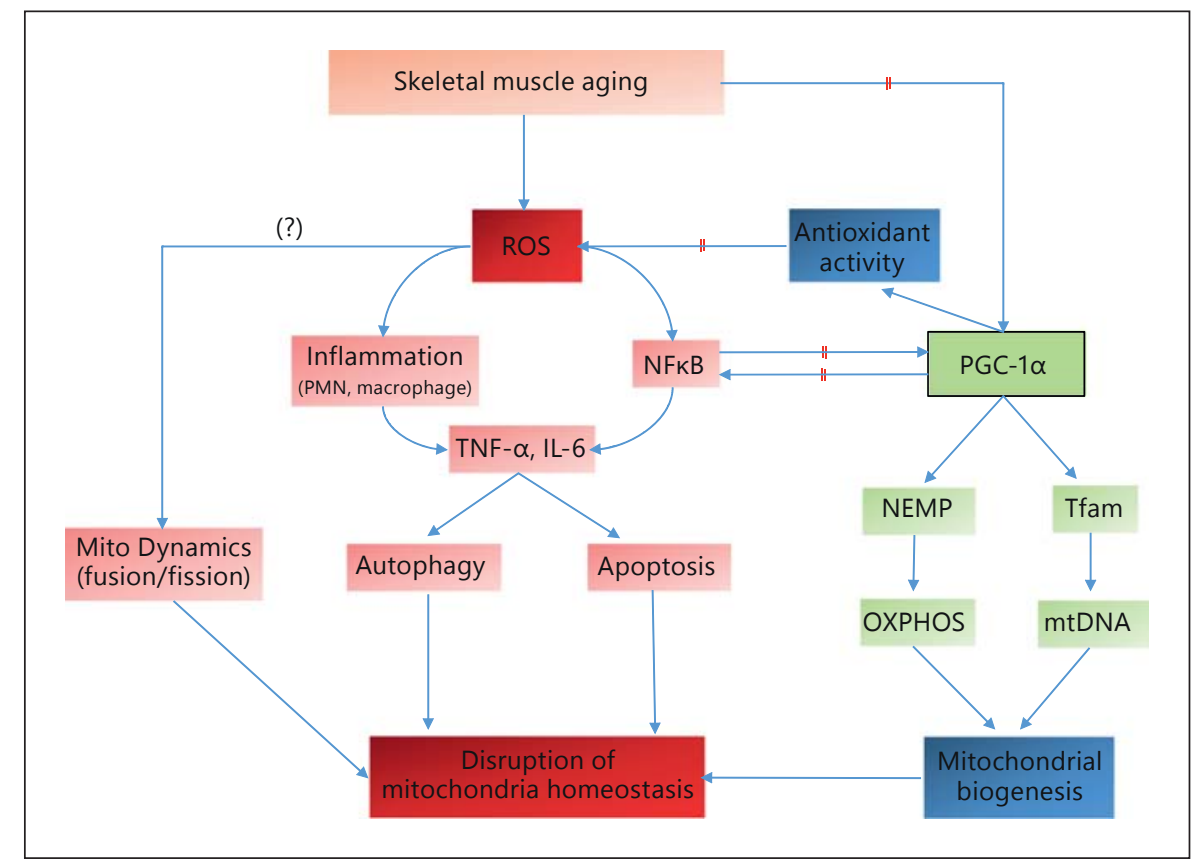

capacity are common muscle pathophysiological characteristics implicated in the development of mitochondrial myopathy. Thus, mitochondrial dysfunction in the etiology of sarcopenia has been extensively examined $[13,14]$.

The most widespread report on senescent muscle is a decline of aerobic capacity caused by both a loss of mitochondrial population density and oxidative phosphorylation (OXPHOS), reflected by a lower respiratory capacity and a reduction of ATP production [15]. Enzyme activities in the Krebs cycle (such as citrate synthase) and electron transport chain (ETC; such as cytochrome c oxidase), known to decline past middle age in rodents, can lose as much as $50 \%$ at the age of 32 months [9]. Mitochondrial protein markers, such as cytochrome c, also showed substantial loss $(\sim 50 \%)$ in old age [16]. There is evidence that these observations of mitochondrial enzyme profiles are mainly caused by decreased mtDNA and mitochondrial protein expression with aging [15]. However, recent data suggest that decline in mitochondrial biogenesis and turnover may also result in accumulation of old and damaged mitochondria that are not functional $[6,17]$. Aging may compromise the ability in aged muscle to clear the dysfunctional mitochondria (mitophagy) [7]. Age-related mtDNA deletion on specific genomes may play an important role in eliciting detrimental effects not only on energy production, but also other vital mitochondrial functions, such as ROS generation, redox homeostasis and apoptosis [18]. Figure 1 illustrates the effects of aging on skeletal muscle, focusing on the various factors that impact on mitochondrial hemostasis and deterioration.

\section{Role of PGC-1a Pathway in Sarcopenia}

PGC-1a, a key regulator of mitochondria biogenesis, was first identified as a functional activator of the peroxisome proliferator-activated receptor (PPAR) $-\gamma$ in brown adipose tissue. Subsequent research revealed that it is a master transcriptional cofactor participating in almost all aspects of mitochondrial functions, ranging from energy fuel selection, muscle fiber differentiation and transformation, to antioxidant gene expression and structural dynamics $[19,20]$. Intracellular coordination processes of mitochondrial biogenesis include transcription of nuclear genes, import of the nuclear-encoded mitochondrial proteins (NEMP), transcription of and replication of mtDNA. PGC-1 $1 \alpha$ interacts with several nuclear transcription factors, including PPAR family members, nuclear respiratory factor (NRF)-1 and NRF-2, and estrogen-related receptor (ERR)- $\alpha$, as well as myocyte enhancer factor-2 (MEF2), forkhead box protein O (FoxO) 1, and sterol regulatory element-binding protein 1 [21]. As such, PGC- 1 a coactivation of NRF- 1 and NRF- 2 binding to target gene promoters induces the expression of numerous NEMP, as well as mitochondrial transcription 
factor A (Tfam), which directly stimulates mtDNA replication and transcription [19]. In addition, a PGC-1a isoform resulting from splicing of primary PGC-1a (PGC1a4) has been found to stimulate muscle hypertrophy due to its ability to activate the IGF-1-mTOR (mammalian target of rapamycin) pathway and suppress myostatin [22]. The potential implication of this novel class of nuclear cofactor in the maintenance of muscle mass and function cannot be overexaggerated, but there has been no report on how aging might affect PGC-1 $1 \alpha 4$ expression and function.

Besides the well-known effects of PGC-1 $\alpha$ on mitochondrial biogenesis, the role of PGC-1 $\alpha$ in NMJ health has been recently highlighted [4]. It was postulated that denervation develops naturally in aged muscle, constituting a major mechanism for sarcopenia. Age-related decline in PGC-1 a may cause instability of the NMJ and consequently a deterioration of myofiber innervation. This theory was supported by experiments with PGC-1 $1 \alpha$ overexpression that upregulated muscle-specific kinase, a tyrosine kinase [4], and in the transgenic mouse model overexpressing muscle-specific MCK-PGC- $1 \alpha$ that showed ameliorated NMJ function marked by increased acetylcholinesterase and acetylcholine receptor density [9].

\section{Effect of Aging on PGC-1 $\alpha$ and Its Implication}

Despite the fact that aging decreases mitochondrial content and function, the effects of aging on the PGC-1a signaling pathway and mitochondrial biogenesis in skeletal muscle are not fully understood. Several studies using rodent and human subjects attempted to clarify whether PGC- $1 \alpha$ level declines with age and its implication in ageassociated muscle atrophy. Chabi et al. [23] reported a 65 and $25 \%$ reduction of $\mathrm{PGC}-1$ a protein content in soleus (slow-twitch oxidative) and plantaris (mixed) muscle, respectively, in 36- versus 6-month-old rats. Interestingly, Tfam content was increased in the aged rats. Kang et al. [16] showed that both PGC-1a mRNA and protein levels were significantly decreased in the soleus muscle of 26-month-old rats, along with lowered Tfam, cytochrome $\mathrm{c}$, and $\mathrm{mtDNA} / \mathrm{nDNA}$ ratio, suggesting an age-related downregulation of the PGC-1a signaling pathway. In agreement with the above rodent studies, Ghosh et al. [24] found that older ( $>65$ years) human subjects had lower PGC-1 $\alpha$ mRNA levels, as well as decreased protein content of NFR-1 and Tfam, in the vastus lateralis (VL) muscle compared to young subjects (18-30 years old). Direct evidence supporting a crucial role of PGC-1a in aging muscle came from a study by Wenz et al. [9]. With a MCK-PGC-1 $\alpha$ transgenic mouse model, the authors demonstrated that muscle-specific overexpression of PGC-1a could ameliorate a wide range of age-related physiological and cellular deteriorations, such as insulin resistance, body fat accumulation, NMJ integrity and systemic inflammation. Importantly, lean body mass was increased comparing MCK-PGC-1 1 versus wild-type mice, indicating that PGC-1 $\alpha$ has a direct role in preserving muscle mass. In contrast to the aforementioned findings, Lanza et al. [25] showed no age difference in the protein levels of PGC-1 $\alpha$, NRF-1 or Tfam in the VL muscle between old (59-76 years) and young (18-30 years) subjects. One potential explanation for the discrepancy of age effects on PGC-1 $a$ signaling could be the different methods with which the data were normalized, ranging from GAPDH and actin (general cytosolic housekeeping genes) to histone $\mathrm{H} 2 \mathrm{~B}$ (a nuclear protein marker), or unspecified. Since aging has a profound impact on the expression of these reference genes, the true age differences might be masked with the different normalization methods.

\section{Potential Mechanisms for Age-Related Decline of \\ PGC-1 $\alpha$ Signaling}

The exact mechanism for age-related decline of PGC1 a signaling is still unclear. However, aging is known to alter several important upstream enzymes and transcription factors that control PGC- $1 \alpha$ expression and posttranslational modification. The findings that aged muscle was not as responsive to cold or thyroid hormone stimulation as the young muscle indicated that upstream signaling pathways could be interrupted with muscle aging [26]. One of such regulators is CREB (cAMP response element-binding protein) binding, a mandatory step for PGC- $1 a$ transactivation. It has been shown that the phospho-CREB to CREB ratio was significantly lower in the aged muscle, along with a dramatically reduced DNA binding [16]. Among the enzymes that control CREB phosphorylation are p38 mitogen-activated protein kinase (p38 MAPK), AMP-activated kinase (AMPK), calcium/calmodulin-dependent protein kinase (CaMK) and calcineurin A, which all could be potential targets for agerelated influences. There have been conflicting findings as to whether the p38 MAPK level is compromised with aging, with both an increase and no change being reported $[27,28]$. Also, some investigators have reported a decrease in muscle AMPK protein level [16], as well as decreased AMPK phosphorylation with aging [29]. The problem with AMPK being a major controller of PGC-1 $\alpha$ is that the enzyme is affected by many intrinsic factors, such as ROS, energy state (AMP/ATP) and other compounds, such as AICAR, and that AMPK also activates 


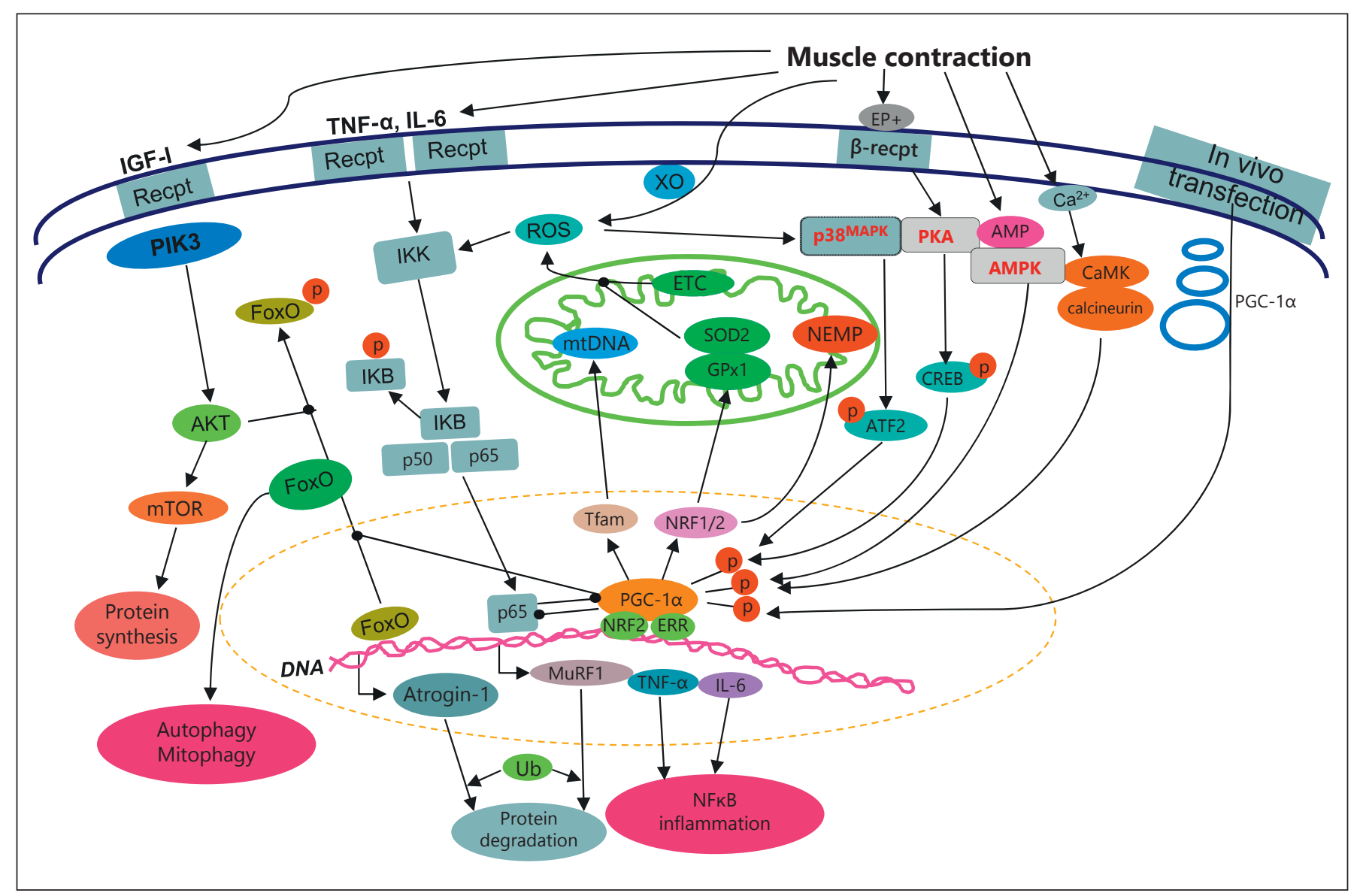

Fig. 2. Schematic illustration of the major inputs for controlling PGC-1 $\alpha$ gene expression and the role of PGC-1 $\alpha$ in controlling the gene expression of negative regulators of muscle protein homeostasis in the muscle cell, and the impact of muscle contraction. The illustration does not include all components and pathways, focus- ing instead on those mentioned in the text. Atrogin-1 is a musclespecific F-box protein, and P50 and P65 are subunits of NFkB. $\mathrm{EP}=$ Epinephrine; IKK = I $\mathrm{KB}$-activated kinase; $\mathrm{PKA}=$ protein $\mathrm{ki}$ nase $\mathrm{A} ; \mathrm{XO}=$ xanthine oxidase. numerous enzymes, making it difficult to conclude its real role in PGC-1 1 a regulation.

Another important mechanism of PGC-1a regulation is redox regulation. ROS generation is increased in aged skeletal muscle that could activate the redox-sensitive signaling pathway nuclear factor $(\mathrm{NF}) \mathrm{\kappa B}$, a negative regulator of PGC-1a. Alvarez-Guardia et al. [30] reported that the p65 subunit of $\mathrm{NF \kappa B}$ is constitutively bound to PGC- $1 \alpha$ in mouse heart, and that NFkB activation by tumor necrosis factor (TNF)- $\alpha$ exposure increases this binding. Conversely, PGC- $1 \alpha$ and PGC- $1 \beta$ can both reduce phosphorylation of NFkB subunit $\mathrm{p} 65$ and weaken its transcriptional potential [31]. However, ROS is also known to activate AMPK and $\mathrm{p} 38$, which might activate PGC- $1 \alpha$ and thus compensate for the downregulation with age [3]. Figure 2 illustrates the potential regulators of the PGC-1 $\alpha$ pathway and its re-

Role of PGC-1 $\alpha$ in Sarcopenia: Etiology and Potential Intervention lationship with the NFkB pathway, and how the interactions may affect muscle protein synthesis and degradation.

Finally, the NAD-dependent deacetylase SIRT1 can be a potential regulator of the PGC- $1 \alpha$ transcriptional activity. As a member of the sirtuin family of proteins, SIRT1 is implicated in a wide range of functions, including cellular differentiation, neural- and cardioprotection, skeletal muscle metabolism and aging [32]. SIRT1 has been reported to directly affect PGC- $1 \alpha$ transcriptional activity by physical interaction, deacetylating and activating PGC-1 1 both in vitro and in vivo [33]. Thus, SIRT1 acts as a sensor that directly detects metabolic perturbations and regulates transcriptional outputs [34]. As animals age, there is a shift of intracellular metabolic status (the $\mathrm{NAD}^{+}: \mathrm{NADH}$ ratio) and redox status that can affect SIRT 1 activity and/or expression. SIRT 1 protein content 
was reported to increase in the skeletal muscle of old compared to young rats [35]. This change might reflect a compensatory need to correct an age-related decline of PGC-1a, but this cannot be concluded. On the other hand, aging has been shown to decrease the expression of SIRT3, a mitochondrial analog of SIRT1, in human skeletal muscle, and this was interpreted as a potential mechanism for the downregulation of mitochondrial biogenesis with age [25]. Taken together, the overall role of SIRT family proteins in mitochondrial function and sarcopenia could be a potentially important one, but requires further investigation.

\section{Crosstalk between PGC-1 $a$ and Other Signaling Pathways}

Role of PGC-1 $\alpha$ in Oxidative Stress and Inflammation

PGC-1 $\alpha$ appears to regulate the expression of endogenous antioxidant proteins, such as superoxide dismutase (SOD) and glutathione peroxidase (GPx) [20]. PGC-1 $\alpha$ knockout (KO) mice exhibits reduced mRNA levels of SOD1 (CuZn-SOD), SOD2 (Mn-SOD), and/or GPx1 and 2, as well as SOD2 protein content, whereas PGC-1a-overexpressed mice showed an upregulation of SOD2 protein content in skeletal muscle [9]. PGC-1 $\alpha$ KO fibroblasts exhibit a decrease in SOD2, catalase and GPx1 mRNA contents compared to wild-type fibroblasts, and PGC-1a KO mice were more vulnerable to oxidative stress [20]. In addition, PGC- $1 \alpha$ has been shown to regulate RNA expression of UCP 2 and 3 in muscle cells [36], suggesting that PGC-1 $1 \alpha$ may also increase the uncoupling capacity and in turn reduce mitochondrial ROS production.

It is well known that aged muscles undergo a chronic, low-grade inflammation marked by increased expression of proinflammatory cytokines, iNOS and C-reactive proteins, which have a significant impact on mitochondrial function, apoptosis and protein degradation that all contribute to sarcopenia [5]. PGC-1a has been postulated to exert anti-inflammatory effects due to its inhibitory effects on FoxO3, inflammatory cytokine expression and its stimulation on antioxidant enzyme expression and vascularization [37]. Thus, PGC-1a KO mice showed elevated TNF- $\alpha$ and interleukin (IL)- 6 in skeletal muscle, as well as higher serum IL- 6 levels than wild-type. Conversely, PGC-1 $\alpha$-overexpressed mice demonstrated lower levels of TNF- $\alpha$ and IL- 6 mRNA, and reduced age-associated increases in TNF- $\alpha$ and IL-6 protein in skeletal muscle [9]. These data support the notion that PGC-1 1 performs protective roles against oxidative stress and inflammation, two major etiological cellular events in aging skeletal muscle.

\section{PGC-1 $\alpha$ and Muscle Proteolysis, Apoptosis and} Autophagy

Sarcopenia is caused by a small but consistent imbalance between muscle protein synthesis and degradation [12]. Two distinct protein degradation pathways exist in skeletal muscle, regulated by different signals and under different physiological and pathological conditions. The ubiquitin-proteasomal pathway is constitutively operative in normal muscle cells and responsible for the turnover of most soluble and myofibrillar muscle proteins. The activity of this pathway is markedly increased in atrophying muscle due to transcriptional activation of ubiquitin, several proteasomal subunit genes, and two muscle-specific ubiquitin ligases, atrogin-1/MAFbx and MuRF1 (muscle RING-finger protein-1), which are induced several-fold during the early stages of muscle atrophy $[38,39]$.

When muscles age, signals from the IGF-AKT-mTOR axis that normally stimulates protein synthesis diminish, resulting in more FoxO3 in the dephosphorylated state and sequestered within the nucleus. Foxo3 is known to be a strong activator of atrogen-1, leading to protein degradation. FoxO3 is also known to downregulate antioxidant enzymes and increase ROS generation, which subsequently activates NFkB. NFkB induces MuRF-1, as well as proinflammatory cytokines such as TNF- $\alpha$, IL- 1 and IL-6, positively feeding forward to enhance proteolysis (fig. 2). On the other hand, PGC- $1 \alpha$ and PGC- $1 \beta$ reduce protein breakdown by inhibiting the transcriptional activity of FoxO3 and NFkB without affecting protein synthesis [39]. Indeed, experimental elevation of muscle PGC-1 $1 \alpha$ levels with either transgenic mice or transfecting myocytes has been shown to attenuate muscle wasting and sarcopenia $[9,40]$.

Autophagy is a catabolic intracellular repair and renewal process, which sequesters and degrades intracellular components through delivery to the degradative organelle, the lysosomal machinery [41]. In skeletal muscle, the FoxO3 transcription factor is necessary and sufficient for the induction of autophagy in skeletal muscle. FoxO3 controls the transcription of autophagy-related genes, including the microtubule-associated protein light chain (LC3) and BCL2/adenovirus E1B 19-kDa-interacting protein 3 (Bnip3) [42]. It becomes increasingly evident that autophagy is a highly selective process and can target specific proteins and organelles, such as mitochondria, via mitophagy. 
When muscle ages, oxidatively damaged proteins increase and, if not efficiently removed, can accumulate, resulting in functional deterioration. Autophagy appears to decline with age in rat skeletal muscle [43]. It has been proposed that loss of autophagy with age leads to the accumulation of damaged mitochondria, which in turn promotes cell death and inflammation [6]. However, Wenz et al. [9] showed that in wild-type mice the LC3-II:LC3-I ratio, a biomarker for increased autophagy, was increased with age, whereas MCK-PGC-1a transgenic mice demonstrated no significant change. The authors concluded that excessive autophagy might be a contributing factor for age-related muscle loss and that PGC-1 $\alpha$ overexpression protected aged mice from excessive degradative processes, such as proteolysis, autophagy and apoptosis. It seems that the role of autophagy in regulating muscle protein turnover requires further clarification.

Muscle aging is not only associated with increased proteolysis, but also with a decreased number of the myocytes [6]. Senescent skeletal muscle undergoes enhanced apoptosis, in which mitochondria play a critical role. All research to date supports the notion that increased apoptotic DNA fragmentation is involved in the development of sarcopenia [44]. However, there is still some controversy regarding whether mitochondrial caspase-dependent pathways dominate the event, as mitochondria-independent pathways, such as cytosolic and nuclear apoptosis-inducing factor and endonuclease $\mathrm{G}$, have also been shown to play a role [5]. PGC-1 1 has been suggested to have antiapoptotic effects and, as such, age-associated decline in PGC-1 1 pathway activity may impact on this degradative process in terms of both muscle fiber size and number.

\section{PGC-1 $\alpha$ and Mitochondrial Dynamics}

Mitochondrial fusion and fission, termed mitochondrial dynamics, can occur independently of general cytosolic protein turnover [4]. PGC-1 has been shown to be involved in mitochondrial network remodeling through controlled fusion and fission. A decline in the ability of fusion to mix damaged and dysfunctional mitochondria with healthy ones due to decreased fusion protein expression may render the muscle cells with greater susceptibility to increased apoptotic tendency in senescent muscle [for details see 7]. Aged muscle shows signs of increased mitochondrial size and reduced metabolic function, and these abnormalities have been attributed to decreased fission and increased fusion [45]. Mitofusion 2 (Mfn2), a key protein for fusion, is regulated by PGC-1a. $\mathrm{H}_{2} \mathrm{O}_{2}$-stimulated $\mathrm{Mfn} 1 / 2$ expression was dramatically attenuated in PGC-1 $\alpha \mathrm{KO}$ nerve cells [20], whereas PGC-1 $\beta$ overexpression markedly enhances Mfn2 mRNA and protein levels in C2C12 myoblasts and myotubes [46].

\section{Effect of Muscle Contraction on PGC-1a: Implication in the Prevention of Sarcopenia}

\section{Skeletal Muscle Disuse Atrophy}

Aging is associated with decreased activity of muscle contraction. There is evidence that many of the cellular events during the development of sarcopenia are similar to those associated with prolonged muscle disuse, such as bed rest, casting and microgravity [6]. Thus, study of muscle immobilization (IM) may provide some useful insights into the mechanism of sarcopenia.

Muscle atrophy caused by prolonged IM appears to be a highly ordered and regulated process which is characterized by decreased muscle fiber cross-sectional area, reduced force production, increased fatigability and insulin resistance [47]. These alterations also include decreased protein synthesis, increased oxidative stress, increased protein degradation and suppression of biogenesis associated with mitochondrial dynamics. Different signaling pathways may be involved in causing muscle atrophy depending on the upstream perturbations, such as decreased IGF1-AKT-mTOR signaling, inflammatory cytokines and NFKB signaling, and reduced nutrition and energy input [39]. During muscle IM, atrophy is associated with a common transcriptional profile and activation of the ubiquitin-proteasome pathway. Imbalance of ROS production and antioxidant defense resulting in oxidative stress plays an important role in protein breakdown in skeletal muscle during periods of inactivity.

A downregulation of PGC-1 1 was observed in muscle atrophy of different models and thought to be a major molecular mechanism for enhanced FoxO phosphorylation, NFaB activation and protein loss [47]. Previous studies suggest that PGC-1 $\alpha$ plays a protective role against protein catabolism and muscle wasting in a variety of contexts. For instance, denervation-induced muscle atrophy and the effects of Duchenne's muscular dystrophy are greatly ameliorated when the amount of PGC-1a is maintained at normal levels or increased [48]. An inactivity-induced deficit of PGC-1 $\alpha$ in skeletal muscle results in a chronic systemic inflammatory state, evidenced by increased TNF- $\alpha$ and IL- 6 expression, and NFkB activation [38]. 
Exercise-Induced Upregulation of the PGC-1 $\alpha$

Pathway

In skeletal muscle, PGC-1a expression is activated during muscle contraction through several potential pathways. CaMKIV and calcineurin A, which are activated through calcium ion dynamics within the muscle, seem to be a direct link [37]. The increased calcium signaling during muscle contraction activates several important transcription factors, such as CREB, a target of CaMKIV, and MEF-2 [49]. Upregulation of PGC-1 $1 \alpha$ expression in response to exercise also involves $\mathrm{p} 38$, which activates MEF-2 and activating transcription factor 2 (ATF2). ATF2-CREB dimerization appears to be an early event in the PGC-1 $\alpha$-mediated signaling processes [49]. In addition to transcriptional activation, p38 also increases PGC- $1 \alpha$ coactivating capacity by phosphorylation in response to cytokine stimulation in muscle cells [50]. Furthermore, as a metabolic energy deprivation sensor, AMPK is activated by exercise due to an increased AMP:ATP ratio and $\mathrm{Ca}^{2+}$ flux during muscle contraction, enhancing PGC-1 1 transcription as well as activity.

Endurance exercise is a powerful stimulus to muscle plasticity and PGC-1 $\alpha$ is a major regulator of exercise-induced phenotypic adaptation and fiber transformation. It is well known that chronic exercise training increases PGC-1 $\alpha$ levels in the skeletal muscle [22, 25, 26, 50-52]. The training adaptation is usually, but not always, accompanied by elevated NRF-1 and Tfam levels. However, endurance training always leads to predictable results in the muscle, such as an elevated mitochondrial respiratory capacity and ATP production, increased expression of the Krebs cycle and ETC enzymes, enhanced fatty acid oxidation and mitochondrial morphological changes [19]. A cross-sectional study showed that endurance-trained subjects had seven times higher PGC-1 $\alpha$, five times higher Tfam and more than 2-fold higher NRF-1 protein contents in VL muscle biopsies than their sedentary counterparts [25]. There is little doubt that the training adaptations were dependent on intact PGC-1 1 signaling pathways, as PGC-1 1 KO mice undergoing endurance training showed virtually no change in mitochondrial markers, such as NRF-1 and cytochrome c content [26]. It is noteworthy that many of the phenotypic changes occurring during endurance training can be demonstrated by a single bout of exercise. For example, Kang et al. [53] reported a 6-fold increase in PGC-1 $\alpha$ content in rat VA muscle after an acute bout of sprinting on a treadmill, along with a $200 \%$ increase in NRF- 1 and Tfam levels. Enhanced CREB and p38 phosphorylation were observed, indicating that upstream pathways controlling PGC- $1 \alpha$ signaling were activated.
Aging Does Not Limit Exercise-Induced Upregulation of $P G C-1 \alpha$

Aging has a profound impact on the adaptability of skeletal muscle to exercise intervention. The majority of the literature agrees that training-induced mitochondrial and metabolic adaptations are not entirely abolished by old age, and that older animals and humans can still benefit from chronic muscular contraction [for a detailed review of this topic, see 2]. This conclusion has been recently confirmed by investigations involving both aged rodents and human subjects $[16,24,25]$. However, it is also widely perceived that aging can attenuate the magnitude of training adaptation seen at a younger age, though a clear explanation of this attenuation is still lacking. In a carefully conducted study, Derbre et al. [26] reported that muscle PGC-1 $\alpha$, NRF- 1 and cytochrome $c$ contents from aged rats did not respond to endurance training as the young rats did, and that the lack of training response was identical to PGC-1 $\alpha$ KO mice. Furthermore, the authors demonstrated that these mitochondrial biogenic markers in aged muscle did not respond to cold exposure or thyroid $\left(\mathrm{T}_{3}\right)$ stimulation, the classic PGC-1a stimulators [19]. It was concluded that an absent response of the PGC-1 a pathway to endurance training might explain the loss of mitochondrial biogenic capacity and metabolic functions seen in the senescent muscle.

In contrast to the above-mentioned findings, we have recently reported clear positive training effects on muscle mitochondrial biogenesis in aged rats [18]. While PGC$1 a$ mRNA and protein levels were 35 and $80 \%$ lower in the soleus muscle of 24- versus 3-month-old rats, respectively, 12 weeks of endurance training resulted in a 2.7 fold higher PGC-1a content in older rats. Training enhanced CREB phosphorylation and its DNA binding capacity, as well as SIRT1 content in aged rats, which were proposed to be potential mechanisms in the observed training adaptation.

Several studies using human subjects provided insightful information with respect to the interactions of aging and exercise training in old age. Ghosh et al. [24] showed that while older subjects had lower muscle PGC-1a mRNA, NRF-1 and Tfam protein contents than young subjects, a 16-week aerobic exercise training program resulted in a more than 2 -fold increase in PGC-1 $\alpha$ mRNA expression and a 1.5-fold increase in NRF- 1 content, but no change in Tfam. Most of the mitochondrial functional markers showed significant improvement despite old age. The authors concluded that 'exercise reverses the phenotype of the old mitochondria'. In a cross-sectional study, Lanza et al. [25] showed that while endurance-trained
Ji/Kang 
young subjects displayed higher mitochondrial protein levels, mtDNA content and PGC-1 $\alpha$ signaling markers (NRF-1, Tfam) than sedentary young subjects, these biomarkers were also significantly higher in trained old subjects compared to their sedentary counterparts, though the magnitude of improvement was consistently lower than in the young subjects. Since the amount of physical exercise these young and older subjects had performed were not matched, there is a possibility that older subjects did less muscular work and thus were exposed to a lower level of stimulus. Alternatively, it is possible that older muscles are less capable of responding to a similar exercise challenge. A study by Iversen et al. [54] has shed some light on this intricacy. Endurance-trained or untrained elderly subjects ( 71 years old) were subjected to an acute bout of bicycle exercise at $75 \%$ of their $\mathrm{VO}_{2 \max }$. Biopsies were obtained from VL muscle before, immediately after and $2 \mathrm{~h}$ after exercise. While both trained and untrained subjects increased PGC- $1 \alpha$ mRNA expression at $2 \mathrm{~h}$, a surprise finding was that untrained subjects displayed a PGC-1 $\alpha$ response that was twice as high ( $\sim 12-$ vs. 6 -fold increase) as the trained subjects. Both groups showed remarkable increases in the phosphorylation level of AMPK and $\mathrm{p} 38$, the two major upstream enzymes that activate
PGC-1 $\alpha$ expression. These findings clearly indicate that the skeletal muscle of elderly subjects maintains the ability to respond to acute exercise and that age should not be the limiting factor of muscle plasticity, at least as far as mitochondrial function is concerned.

\section{Conclusion}

Over the last decade, PGC-1 $\alpha$ has been shown to be the most potent activator of mitochondrial biogenesis and function. This versatile and master transcriptional regulator has received increasing attention among muscle biologists. Undoubtedly, PGC-1 $1 \alpha$ plays a critical role in controlling numerous genes that affect a broad range of muscle morphology and physiological function due to its interactions with other signaling pathways that control mitochondrial homeostasis and protein balance. Inactivity and aging are two important and interconnected negative factors for PGC-1 $\alpha$ gene expression. Thus, increasing stimulation to this pathway through muscle contraction can maintain and enhance the PGC-1a signaling activity and in turn ameliorate other vital functions in the senescent muscle.

\section{References}

1 Metter EJ, Talbot LA, Schrager M, Conwit R: Skeletal muscle strength as a predictor of allcause mortality in healthy men. J Gerontol A Biol Sci Med Sci 2002;57:B359-B365.

2 Ljubicic V, Joseph A, Saleem A, et al: Transcriptional and post-transcriptional regulation of mitochondrial biogenesis in skeletal muscle: effects of exercise and aging. Biochim Biophys Acta 2010;1800:223-234.

-3 Gouspillou G, Picard M, Godin R, Burelle Y, Hepple R: Role of peroxisome proliferative activated receptor gamma coactivator 1 -alpha (PGC-1 $\alpha$ ) in denervation-induced atrophy in aged muscle: facts and hypotheses. Longev Healthspan 2013;2:13.

4 Johnson ML, Robinson MM, Nair KS: Skeletal muscle aging and the mitochondrion. Trends Endocrin Met 2013;24:247-256.

5 Marzetti E, Calvani R, Cesari M, et al: Mitochondrial dysfunction and sarcopenia of aging: from signaling pathways to clinical trials. Int J Biochem Cell Biol 2013;45:22882301.

6 Calvani R, Joseph A, Adhihetty PJ, et al: Mitochondrial pathways in sarcopenia of aging and disuse muscle atrophy. Biol Chem 2013; 394:393-414.

7 Wallace DC: A mitochondrial paradigm of metabolic and degenerative diseases, aging, and cancer: a dawn for evolutionary medi- 15 Short KR, Bigelow ML, Kahl J, et al: Decline cine. Annu Rev Genet 2005;39:359.

8 Finck BN, Kelly DP: PGC-1 coactivators: inducible regulators of energy metabolism in health and disease. J Clin Invest 2006;116: 615-622.

9 Wenz T, Rossi SG, Rotundo RL, Spiegelman BM, Moraes CT: Increased muscle PGC-1a expression protects from sarcopenia and metabolic disease during aging. Proc Natl Acad Sci U S A 2009;106:20405-20410.

${ }_{10}$ Frontera WR, Hughes VA, Fielding RA, Fiatarone MA, Evans WJ, Roubenoff R: Aging of skeletal muscle: a 12-year longitudinal study. J Appl Physiol 2000;88:1321-1326.

11 Kent-Braun JA, Ng AV, Young K: Skeletal muscle contractile and noncontractile components in young and older women and men. J Appl Physiol 2000;88:662-668.

12 Marcell TJ: Sarcopenia: causes, consequences, and preventions. J Gerontol A Bilo Sci Med Sci 2003;58:M911-M916.

13 Kujoth G, Hiona A, Pugh T, et al: Mitochondrial DNA mutations, oxidative stress, and apoptosis in mammalian aging. Science 2005; 309:481-484

14 Chakravarti B, Chakravarti DN: Oxidative modification of proteins: age-related changes. Gerontology 2006;53:128-139. in skeletal muscle mitochondrial function with aging in humans. Proc Natl Acad Sci U S A 2005;102:5618-5623.

16 Kang C, Chung E, Diffee G, Ji LL: Exercise training attenuates aging-associated mitochondrial dysfunction in rat skeletal muscle: role of PGC-1a. Exp Gerontol 2013;48:1343-1350.

-17 Terman A, Gustafsson B, Brunk UT: Mitochondrial damage and intralysosomal degradation in cellular aging. Mol Aspects Med 2006;27:471-482.

18 Marzetti E, Leeuwenburgh C: Skeletal muscle apoptosis, sarcopenia and frailty at old age. Exp Gerontol 2006;41:1234-1238.

19 Wu Z, Puigserver P, Andersson U, et al: Mechanisms controlling mitochondrial biogenesis and respiration through the thermogenic coactivator PGC-1. Cell 1999;98:115-124.

20 St-Pierre J, Drori S, Uldry M, et al: Suppression of reactive oxygen species and neurodegeneration by the PGC-1 transcriptional coactivators. Cell 2006;127:397-408

21 Knutti D, Kralli A: PGC-1, a versatile coactivator. Trends Endocrin Met 2001;12:360-365.

22 Ruas JL, White JP, Rao RR, Kleiner S, et al: A PGC-1 $\alpha$ isoform induced by resistance training regulates skeletal muscle hypertrophy. Cell 2012;151:1319-1331.
Role of PGC-1 1 in Sarcopenia: Etiology and Potential Intervention
Gerontology 2015;61:139-148 DOI: $10.1159 / 000365947$ 
-23 Chabi B, Ljubicic V, Menzies KJ, Huang JH, Saleem A, Hood DA: Mitochondrial function and apoptotic susceptibility in aging skeletal muscle. Aging Cell 2008;7:2-12.

24 Ghosh S, Lertwattanarak R, Lefort N, et al: Reduction in reactive oxygen species production by mitochondria from elderly subjects with normal and impaired glucose tolerance. Diabetes 2011;60:2051-2060.

-25 Lanza IR, Short DK, Raghavakaimal S, Rasu $\mathrm{R}$, et al: Endurance exercise as a countermeasure of aging. Diabetes 2008;57:29332942.

-26 Derbre F, Gomez-Carbrera C, Nascimento $\mathrm{AL}$, et al: Age associated low mitochondrial biogenesis may be explained by lack of response of PGC-1a to exercise training. Age 2012;34:669-679.

-27 Qiang W, Weiqiang K, Qing Z, Pengju Z, Yi L: Aging impairs insulin-stimulated glucose uptake in rat skeletal muscle via suppressing AMPKa. Exp Mol Med 2007;39:535-543.

28 Hornberger TA, Mateja RD, Chin ER, Andrews JL, Esser KA: Aging does not alter the mechanosensitivity of the p38, p70S6k, and JNK2 signaling pathways in skeletal muscle. J Appl Physiol 2005;98:1562-1566.

29 Williamson D, Gallagher P, Harber M, Hollon C, Trappe S: Mitogen-activated protein kinase (MAPK) pathway activation: effects of age and acute exercise on human skeletal muscle. J Physiol (Lond) 2003;547: 977-987.

-30 Álvarez-Guardia D, Palomer X, Coll T, et al: The p65 subunit of NF- $\kappa B$ binds to PGC- $1 \alpha$, linking inflammation and metabolic disturbances in cardiac cells. Cardiovasc Res 2010; 87:449-458.

- 31 Eisele PS, Salatino S, Sobek J, Hottiger MO, Handschin C: The PGC-1 coactivators repress the transcriptional activity of NF- $\mathrm{KB}$ in skeletal muscle cells. J Biol Chem 2013;288: 2246-2260.

32 Dali-Youcef N, Lagouge M, Froelich S, Koehl C, Schoonjans K, Auwerx J: Sirtuins: the 'magnificent seven', function, metabolism and longevity. Ann Med 2007;39:335-345.
Nemoto S, Fergusson M, Finkel T: SIRT1 functionally interacts with the metabolic regulator and transcriptional coactivator PGC1a. J Biol Chem 2005;280:16456-16460.

34 Koltai E, Szabo Z, Atalay M, et al: Exercise alters SIRT1, SIRT6, NAD and NAMPT levels in skeletal muscle of aged rats. Mech Ageing Dev 2010;131:21-28.

-35 Rodgers JT, Lerin C, Gerhart-Hines Z, Puigserver P: Metabolic adaptations through the PGC-1 $\alpha$ and SIRT1 pathways. FEBS Lett 2008;582:46-53.

36 St-Pierre J, Lin J, Krauss S: Bioenergetic analysis of peroxisome proliferator-activated receptor $\gamma$ coactivators $1 \alpha$ and $1 \beta$ (PGC- $1 \alpha$ and PGC-1 $\beta$ ) in muscle cells. J Biol Chem 2003; 278:26597-26603.

37 Handschin C, Spiegelman BM: The role of exercise and PGCla in inflammation and chronic disease. Nature 2008;454:463-469.

38 Kang C, Ji LL: Muscle immobilization and remobilization downregulates PGC-1 $\alpha$ signaling and the mitochondrial biogenesis pathway. J Appl Physiol 2013;115:1618-1625.

39 Sendri M: Signaling in muscle atrophy and hypertrophy. Physiology 2008;23:160-170.

40 Geng T, Li P, Yin X, Yan Z: PGC-1a promotes nitric oxide antioxidant defenses and inhibits FOXO signaling against cardiac cachexia in mice. Am J Path 2011;178:1738-1748.

41 Yorimitsu T, Klionsky DJ: Autophagy: molecular machinery for self-eating. Cell Death Differ 2005;12:1542-1552.

42 Mammucari C, Milan G, Romanello V, et al: FoxO3 controls autophagy in skeletal muscle in vivo. Cell Metab 2007;6:458-471.

43 Green DR, Galluzzi L, Kroemer G: Mitochondria and the autophagy-inflammation-cell death axis in organismal aging. Science 2011; 333:1109-1112.

44 Marzetti E, Hwang JC, Lees HA, et al: Mitochondrial death effectors: relevance to sarcopenia and disuse muscle atrophy. Biochim Biophys Acta 2010;1800:235-244.
45 Yoon YS, Yoon DS, Lim IK, et al: Formation of elongated giant mitochondria in DFO-induced cellular senescence: involvement of enhanced fusion process through modulation of Fis1. J Cell Physiol 2006;209:468-480.

46 Liesa M, Borda-d'Água B, Medina-Gómez G, et al: Mitochondrial fusion is increased by the nuclear coactivator PGC-1 $\beta$. PLoS One 2008; 3:e3613.

47 Kandarian SC, Jackman RW: Intracellular signaling during skeletal muscle atrophy. Muscle Nerve 2006;33:155-165

48 Hanai JI, Cao P, Tanksale P, Imamura S, Koshimizu E, Zhao J, Kishi S, et al: The musclespecific ubiquitin ligase atrogin-1/MAFbx mediates statin-induced muscle toxicity. J Clin Invest 2007;117:3940-3951.

49 Vercauteren K, Pasko RA, Gleyzer N, Marino VM, Scarpulla RC: PGC-1-related coactivator: immediate early expression and characterization of a CREB/NRF-1 binding domain associated with cytochrome $c$ promoter occupancy and respiratory growth. Mol Cell Biol 2006;26:7409-7419.

50 Akimoto T, Pohnert SC, Li P, et al: Exercise stimulates $P g c-1 \alpha$ transcription in skeletal muscle through activation of the p38 MAPK pathway. J Biol Chem 2005;280:19587-19593.

51 Gordon JW, Rungi AA, Inagaki H, Hood DA Effects of contractile activity on mitochondrial transcription factor A expression in skeletal muscle. J Appl Physiol 2001;90:389396.

52 Feng H, Kang C, Dickman JR, et al: Traininginduced mitochondrial adaptation: role of peroxisome proliferator-activated receptor $\gamma$ coactivator- $1 \alpha$, nuclear factor $-\kappa B$ and $\beta$-blockade. Exp Physiol 2013;98:784-795.

53 Kang C, O’Moore KM, Dickman JR, Ji LL: Exercise activation of muscle peroxisome proliferator-activated receptor- $\gamma$ coactivator- $1 \alpha$ signaling is redox sensitive. Free Radical Bio Med 2009;47:1394-1400.

54 Iversen N, Krustrup P, Rasmussen H, et al: Mitochonddrial biogenesis and angiogenesis in skeletal muscle of the elderly. Exp Gerontol 2011;46:670-678. 